\title{
Abortion Caused by Coxiella burnetii in a Cow and Goat in Korea
}

Kyung-Hyun Lee ${ }^{1}$, Hyun-Kyoung Lee ${ }^{1}$, Kang-Hyun Baek ${ }^{1}$, Jae-Gu Oem ${ }^{1}$, Ha Young Kim ${ }^{1}$, Lucienvan Keulen ${ }^{2}$, Jae-Won Byun ${ }^{1}$, Byung-Jae So ${ }^{1}$ and Eun-Jin Choi $^{1^{*}}$

${ }^{1}$ Animal Disease Diagnostic Division, Animal and Plant Quarantine Agency, 175 Anyang-ro, Manan-gu, Anyang 430-757, Korea

${ }^{2}$ Central Veterinary Institute, Wageningen University and Research Centre, PO Box 65,8200 AB Lelystad, The Netherlands

*Corresponding author: Eun-Jin Choi, Animal Disease Diagnostic Division, Animal and Plant Quarantine Agency, 175 Anyang-ro, Manan-gu, Anyang 430-757, Korea, Tel: +82-54-912-0461; Fax: +82-31-467-0465; E-mail: mylovehyun@korea.kr

Rec date: February 14, 2018; Acc date: February 21, 2018; Pub date: February 23, 2018

Copyright: @ 2018 Lee $\mathrm{KH}$, et al. This is an open-access article distributed under the terms of the Creative Commons Attribution License, which permits unrestricted use, distribution, and reproduction in any medium, provided the original author and source are credited.

\begin{abstract}
$Q$ fever is a zoonosis caused by Coxiella burnetii, an obligate intracellular Gram-negative bacterium. $Q$ fever is usually subclinical, but is occasionally associated with abortion in ruminants. Two cases of abortion, one in a goat and the other in a cow, were reported to the Animal and Plant Quarantine Agency for diagnostic testing. Histologically, suppurative placentitis was apparent in both cases and more severe in the goat than in the cow. $C$. burnetii antigens were detected by polymerase chain reaction of DNA from various tissue extracts and antibodies were detected in the sera in both cases. To our knowledge, this is the first report of bovine and caprine abortion caused by $C$. burnetii in South Korea.
\end{abstract}

Keywords: Coxiella burnetii; Bovine; Caprine; Korea; Abortion

\section{Introduction}

Coxiella burnetii, an obligate intracellular Gram-negative bacterium, is the pathogen that causes $\mathrm{Q}$ fever in humans and animals. This bacterium causes a variety of symptoms, including acute flu-like symptoms, pneumonia, hepatitis and chronicendocarditis in humans [1]. C. burnetii infection in animals is generally asymptomatic, but can become symptomatic in pregnancy. Metritis, abortion, stillbirth, and delivery of weak offspring are the most frequent clinical signs of the disease [2]. In symptomatic cases, C. burnetii is excreted via feces, vaginal mucus, milk, and birth products. In pregnant goats, the clinical manifestation of $C$. burnetii infection is abortion or stillbirth in the final stage of gestation [3-5]. Routine diagnosis of Q fever in cattle is usually based on a specific enzyme-linked immunosorbent assay or complement fixation test [4]. Culturing the bacterium is difficult and requires a Biosafety Level 3 laboratory. Several safe and useful polymerase chain reaction (PCR) methods are currently available for detecting $C$. burnetii infection in milk, feces and vaginal mucus, and in aborted bovine fetuses and placental tissue $[1,6,7]$. C. burnetii is found in trophoblasts and mononuclear cells in an infected placenta [8]. $C$. burnetii is present worldwide, with the exception of New Zealand [6]. However, abortion caused by $C$. burnetii in cattle and goats has not been reported in Korea. In this study, we describe the first known cases of bovine and caprine abortion caused by $C$. burnetii in this country.

\section{Histories}

An aborted fetus and placenta from a Holstein cow were submitted to the Animal and Plant Quarantine Agency on July 15, 2013. The cow was from a farm that housed approximately 150 cows in Hawseong, Gyeonggido, Korea. The cows were vaccinated against foot and mouth disease in early 2013. Abortion samples, including the fetus, placenta, and blood from a Korean black goat were subsequently submitted on May 14, 2014. The goat was one of approximately 1,000 goats raised on a farm in Imsil, Jeonrabukdo, Korea. Abortion at various gestational ages was detected in 30 goats on this farm between March and May 2014; all goats were treated with penicillin after abortion from March 2014 onwards, but the incidence was not reduced.

\section{Post-mortem findings}

Necropsy was performed on both fetuses and placentas. All tissues were fixed in $10 \%$ buffered formalin and processed normally. Tissue sections $4-\mu \mathrm{m}$-thick were obtained from multiple organs for hematoxylin and eosin staining and immunohistochemistry. All organs were fixed in formalin, embedded in paraffin, sectioned at $4-\mu \mathrm{m}$ thickness, and stained with hematoxylin and eosin for microscopic examination. Immunohistochemistry was performed using the $\mathrm{ABC}$ method. Antigen retrieval was performed in citrate solution ( $\mathrm{pH} 6.0)$ and anti- $C$. burnetii (MAB 313) was used as the primary antibody. For microbiology, the fetus and placenta were inoculated onto Brucella and blood agar, and then incubated for 3 days in a $5 \% \mathrm{CO}_{2}$ incubator. Multiplex PCR was carried out to detect bacterial agents such as Neospora canis, Leptospira spp, Campylobacter fetus, Listeria monocytogenes, and C. burnetii. PCR was also used to detect viral pathogens such as bovine herpesvirus types 1 and 5, bovine viral diarrhea virus, Aino virus, Chuzan virus, Ibaraki virus, bovine ephemeral fever virus, and Akabane virus. Both organs were examined for bacterial and viral pathogens.

The crown-rump lengths in the cow and goat were $35 \mathrm{~cm}$ and 30 $\mathrm{cm}$, respectively. Autolysis was observed in the fetus and placenta in both cases. The placental cotyledons were hemorrhagic in the caprine case.

Mild suppurative placentitis was found in the bovine placenta and severe suppurative placentitis and mineralization were found in the caprine placenta (Figure 1). It was difficult to identify a histologic lesion that would have caused autolysis in the bovine placenta; however, the goat placenta contained multifocal to confluent areas of mineralization and diffuse suppurative placentitis with heavy neutrophil infiltration and extensive necrosis of the cotyledonary villi and intercotyledonary epithelium. 


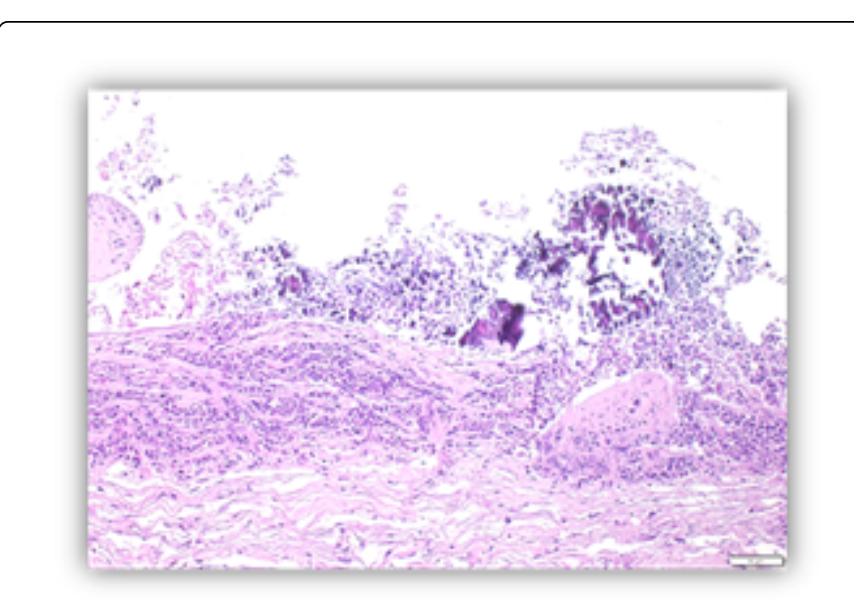

Figure 1: Severe infiltration of polymorphonuclear leukocytes and mononuclear cells in the chorionic stroma with foci of mineralization in the placenta of a goat with Coxiella burnetii infection. Bar=50 $\mu \mathrm{m}$.

No lesions were observed in the fetal organs. The bovine and caprine placentas were positive for $C$. burnetii on PCR testing. Immunohistochemistry revealed $C$. burnetii antigen in the caprine placenta (Figure 2). The bovine fetus was negative by PCR because of autolysis; however, positive reactions were observed in all internal organs of the caprine fetus. No positive reaction to other viral or bacterial agents was detected. A strong Q fever-positive reaction was detected in the serum of both dams.

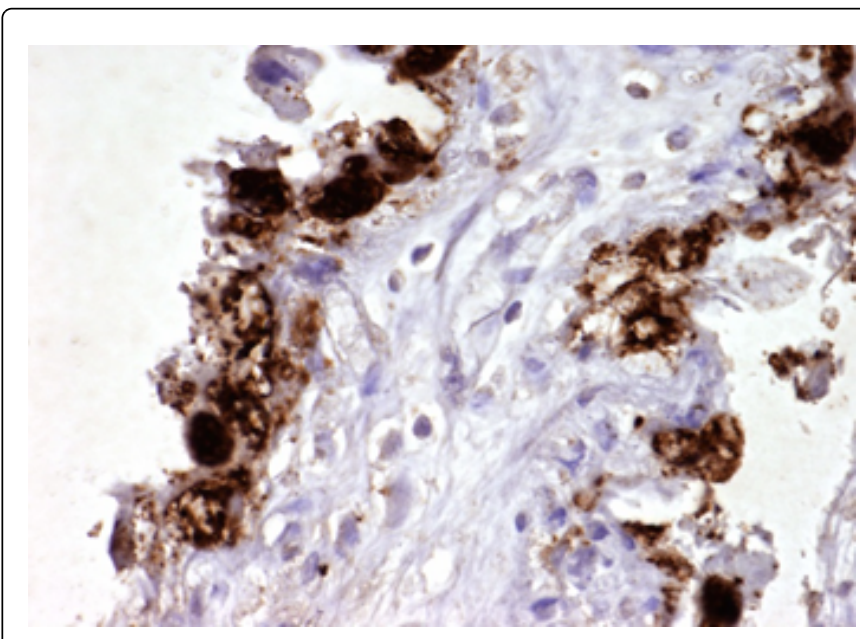

Figure 2: Strong positive reaction (brown) of trophoblasts in the placenta of a goat with Coxiella burnetii infection. Bar $=50 \mu \mathrm{m}$.

\section{Discussion}

Both cases described here were diagnosed as abortion caused by $C$. burnetii on the basis of histopathologic features and a positive PCR test. The life cycle, route of infection, and pathogenesis of $C$. burnetii are under experimental investigation. The major routes of infection in humans are inhalation of contaminated aerosol following parturition of an infected animal and ingestion of contaminated raw milk or milk products [8]. A minor route of infection is through the skin [7].

No gross placental lesions were present in the caprine case reported here, but such lesions have been described in previous cases of bovine coxiellosis [9]. Microscopically, the placentas showed a range of microscopic lesions varying from severe hemorrhage and necrosis to mildly increased numbers of mononuclear cells within a diffusely edematous chorionic stroma. These findings are similar to those described in a previous report on inflammatory changes in the placentas of four cows with mild $C$. burnetii infection [8]. Further, there has been a report of more severe placental lesions in caprine coxiellosis [3].

The seropositivity of $C$. burnetii in Korean dairy cattle and native Korean goats has been reported [9]. C. burnetii seropositivity has also been confirmed in Korean dairy farmers and health professionals, including veterinarians working in local clinics and laboratories [10]. Considering the high rate of $\mathrm{Q}$ fever infection observed among cattle and goats and previous reports of humans with high-risk occupations, such as herders, veterinarians and abattoir workers, contracting this disease, the risk of transmission of $C$. burnetii from livestock to humans is presumed to be high [10].

This report includes the first case of an antigen-positive placental lesion with abnormal pathology in Korea. Further studies of placentas in ruminants are needed to clarify the distribution and prevalence of C. burnetii. Clinicians should consider $C$. burnetii in the differential diagnosis in cases of abortion in animals that have been vaccinated and treated with antibiotics.

\section{Acknowledgments}

This work was supported by a grant from the Animal and Plant Quarantine Agency of Korea (N-AD21-2010-19-04).

\section{Authors' Contributions}

KHL, HKL, and KHB performed the pathologic analysis. EJC and BJS designed the general experiments. HYK and JWB performed the microbiologic analysis. LVK performed the immune histochemical analysis. All authors interpreted the data, critically revised the manuscript for important intellectual content, and approved the final version of the manuscript.

\section{References}

1. Pritchard GC, Smith RP, Errington J, Hannon S, Jones RM, et al. (2011) Prevalence of Coxiella burnetii in livestock abortion material using PCR. Vet Rec 169: 391.

2. Berri M, Rekiki A, Boumedine KS, Rodolakis A (2009) Simultaneous differential detection of Chlamydophila abortus, Chlamydophila pecorum and Coxiella burnetii from aborted ruminant's clinical samples using multiplex PCR. BMC Microbiol 9: 130-137.

3. Berri M, Rousset E, Champion JL, Russo P, Rodolakis A (2007) Goats may experience reproductive failures and shed Coxiella burnetii at two successive parturitions after $Q$ fever infection. Res Vet Sci 83: 47-52.

4. Berri M, Rousset E, Hechard C, Champion JL, Dufour P, et al. (2005) Progression of $\mathrm{Q}$ fever and Coxiella burnetii shedding in milk after an outbreak of enzootic abortion in a goat herd. Vet Rec 156: 548-549.

5. Hatchette T, Campbell N, Hudson R, Raoult D, Marrie TJ (2003) Natural history of $\mathrm{Q}$ fever in goats. Vector Borne Zoonotic Dis 3: 11-15. 
Citation: Lee KH, Lee HK, Baek KH, Oem JG, Kim HY, et al. (2018) Abortion Caused by Coxiella burnetii in a Cow and Goat in Korea. J Vet Sci Technol 9: 516. doi:10.4172/2157-7579.1000516

Page 3 of 3

6. Jones RM, Twomey DF, Hannon S, Errington J, Pritchard GC, et al. (2010) Detection of Coxiella burnetii in placenta and abortion samples from British ruminants using real-time PCR. Vet Rec 167: 965-967.

7. Monno R, Fumarola L, Trerotoli P, Cavone D, Giannelli G, et al. (2009) Seroprevalence of Q fever, brucellosis and leptospirosis in farmers and agricultural workers in Bari, Southern Italy. Ann Agric Environ Med 16: 205-209.

8. Bildfell RJ, Thomson GW, Haines DM, McEwen BJ, Smart N, et al. (2000) Coxiella burnetii infection is associated with placentitis in case of bovine abortion. J Vet Diagn Invest 12: 419-425.
9. Lorenz H, Jäger C, Willems H, Baljer G (1998) PCR detection of Coxiella burnetii from different clinical specimens, especially bovine milk, on the basis of DNA preparation with silica matrix. Appl Environ Microbiol 64: 4234-4237.

10. Jung BY, Seo MG, Lee SH, Byun JW, Oem JK, et al. (2014) Molecular and serologic detection of Coxiella burnetii in native Korean goats (Capra hircus coreanae). Vet Microbiol 17: 152-155. 